\title{
Role of molecular studies in the diagnosis of lung adenocarcinoma
}

\author{
Samuel A Yousem \\ Department of Pathology, University of Pittsburgh Medical Center-Presbyterian Campus, Pittsburgh, \\ $P A, U S A$
}

\begin{abstract}
Molecular alterations in adenocarcinoma of the lung have resulted in new therapeutic options for treatment of high-stage disease. Such changes are usually mutually exclusive and can be documented in small specimen samples. Most analyses are DNA-based, utilizing sequencing or fluorescence in situ hybridization to observe amplifications or translocations. Particular success in theranostics has focused on epidermal growth factor receptor (EGFR), anaplastic lymphoma kinase gene (ALK), and BRAF gene changes, each allowing personalized therapies. Interestingly, these molecular changes have correlated with distinct, although not unique, demographics, histopathologies, and response to pharmacological agents.

Modern Pathology (2012) 25, S11-S17; doi:10.1038/modpathol.2011.156
\end{abstract}

Keywords: adenocarcinoma; ALK gene; BRAF; EGFR; KRAS; lung; mutation

Non-small cell lung cancer (NSCLC) is a major cause of death worldwide, with most patients being diagnosed with disease at an advanced stage when treatment is only palliative. As a consequence, great interest has focused on new therapeutic options for its treatment, and the most recent efforts have focused on attacking markers of molecular alterations in lung cancer. Although the varieties of molecular changes are infinite, a subset of carcinomas has evolved that have specific, rather 'simple,' genetic mutations that have offered some positive therapeutic interventions. ${ }^{1}$ This increase in molecular understanding has been highlighted by somatic mutations in epidermal growth factor receptor (EGFR), where treatment of EGFR kinase inhibitors in EGFR-mutated NSCLC leads to a superior response rate, a prolonged progression-free survival, and improved quality of life as compared with conventional cytoxic chemotherapy. ${ }^{2-10}$ In this rapidly evolving field, two other molecular changes have been identified, the first being fusion of the anaplastic lymphoma kinase gene $(A L K)$ with the echinoderm microtubule-associated protein-like 4 (EML4) and the identification of BRAF mutations in

Correspondence: Dr SA Yousem, MD, Department of Pathology, University of Pittsburgh Medical Center-Presbyterian Campus, Presbyterian Campus-C620, 200 Lothrop Street, Pittsburgh, PA 15213-2582, USA.

E-mail: yousemsa@upmc.edu

Received 11 August 2011; accepted 26 August 2011 lung cancer, where the most common mutation of a valine to glutamate substitution at Codon 30 (V600E) results in a potential response to new chemotherapeutic agents such as PLX4032, associated with an $80 \%$ response rate in advanced-stage malignant melanoma with BRAF-V600E mutations. This brief review will focus on these three genes and a surgical pathology approach to their study.

\section{Molecular testing}

Molecular testing in lung carcinoma needs to be first assessed in a broad context. ${ }^{11}$ First, the literature clearly shows that smoking- and non-smokingassociated lung carcinomas are characterized by hundreds of mutations, and that these mutations change over time, often in response to chemoradiation interventions. Most therapeutic successes have been achieved in low mutation settings, ie, nonsmokers, with very unique mutations in the lung carcinoma. Second, most successes have been in non-small cell carcinomas, and almost exclusively adenocarcinoma or large cell carcinomas, and where the genetic mutations are exclusive of one another. Specifically in this review, EGFR, ALK, and B-RAF genetic changes occur individually in the absence of alterations of the other. Third, most studies have looked at DNA abnormalities. The correlation with DNA expression, RNA changes, and proteomics is still in its infancy. Finally, at present, therapeutic successes have been restricted to high-stage or 
recurrent disease. ${ }^{4}$ The role of such studies in the setting of resectable disease is under investigation.

At present, the molecular testing in lung cancer has focused largely on non-small cell carcinoma, specifically adenocarcinoma, and those poorly differentiated non-small cell carcinomas such as large cell carcinoma and its variants. For the most part, molecular testing for therapeutic decision-making for squamous cell carcinoma, small cell carcinoma, and low-grade neuroendocrine tumors, eg, carcinoid tumors has not become routine, although certainly in the future, new therapeutic options will drive molecular testing in this subset. Molecular testing in NSCLC can be done on small tissue samples, including small core, endoscopic, and fine-needle biopsies and cytology specimens. ${ }^{12,13}$ These specimens can be enriched for tumor cells, as somewhere between 200 and 400 microdissected cells allow testing to be performed with good sensitivity and specificity. Almost all molecular tests can be performed on formalin-fixed paraffin-embedded tissue, and therefore, biopsies and cell blocks are best processed with this fixative rather than others that interfere with such testing, eg, Bouin's, Zenker's, decalcification solutions. As these biopsy and cytology specimens have only small numbers of tumor cells, it is recommended that when lung carcinoma is a significant consideration, at primary processing, a significant number of unstained slides should be cut so that microdissected tissue sections can be eluted from these unstained slides for future molecular testing. In this setting, great sensitivity has been achieved using primarily DNA-based assays. Although intratumoral heterogeneity has been questioned in the past, most recent studies show that heterogeneity is minimal (approximately $10 \%) .{ }^{13}$ Nonetheless, it does raise questions as to the appropriate specimen to use for molecular assays. For example, should one assay tissue from an original resectioned specimen or a biopsy of a subsequent recurrence. The answer to this question is unclear; although recognizing that malignancies progress and evolve, this author believes that if one can obtain a sample of the recurrence, it would provide the best molecular information on response to interventional therapies. Discordances in results between primary tumors and their metastatic foci varying between $11-50 \%$ (depending on the gene under investigation and the assay performed) have been observed. ${ }^{12-16}$ Therefore, it is likely preferable to assay the metastasis, recurrence, or post-treatment sample. ${ }^{17}$

\section{EGFR alterations and lung adenocarcinoma}

EGFR is a receptor tyrosine kinase of the HER family, which consists of four closely related receptors. This $170 \mathrm{kDa}$ membrane-bound protein has both an extracellular membrane and intracellu- lar component, and has approximately 28 exons on chromosome $7 \mathrm{p} 21$. The receptor is involved in cell signaling, and activation of the receptor primarily results in augmentation of cell proliferation, inhibition of apoptosis, and increased angiogenesis. The two most common EGFR mutations are short in-frame deletions of exon 19, usually of $15 \mathrm{bp}$, and a point mutation (CTG2CGG) in exon 21, at nucleotide 2573 , which result in a substitution of leucine by arginine at Codon 858 (L858R). Together, these two mutations account for approximately $90 \%$ of EGFR mutations in lung adenocarcinoma.

EGFR mutations were first identified in adenocarcinomas arising in non-smoking individuals. Subsequent studies indicated a significant rate of EGFR mutations in young non- or light-smoking female East Asians, particularly from Japan, Indonesia, and Korea, who presented with low-stage disease. ${ }^{17-19}$ In these countries, EGFR mutations in this patient population had an incidence of $30-50 \%$, but when subsequent studies were done in Western population, the rates were approximately half of this number, somewhere between 10 and $20 \%$ in most studies, but with similar demographics. ${ }^{2-10}$ Such mutations were particularly appealing to interventionalists, because specific EGFR tyrosine kinase inhibitors (Gefitinib and Erlotinib) offered interventions and positively affected progressionfree survival in high-stage patients. Recent phase III trials in lung adenocarcinoma with EGFR mutations have demonstrated that EGFR-mutated lung cancers have better treatment outcomes in the form of response rate and progressive-free survival when treated with EGFR-specific inhibitors.

With the development of molecular testing, an attempt to establish histomolecular correlations has been appealing. In EGFR-mutated adenocarcinomas, there are suggestive, but not absolute associations with well-differentiated adenocarcinoma. In fact, a theme of this discussion of molecular alterations in lung adenocarcinoma spectrum is that the histological variations of NSCLC, especially adenocarcinomas, that display a molecular mutation is significant enough that such testing should be done in all lung adenocarcinomas rather than simply being restricted to those that have the most suggestive histopathology; EGFR-mutated adenocarcinomas are typically adenocarcinomas of low stage that have a prominent lepidic growth pattern, where this lepidic growth pattern is associated with acinar or papillary growth. ${ }^{18,20}$ (Figures 1-5) Such adenocarcinomas typically do not have evidence of necrosis and tend to be associated with a minimal host stromal and immune response. ${ }^{18,21}$ These adenocarcinomas show an immunophenotype of cells of the terminal reserve unit, with antigen expression characteristic of Clara and Type II alveolar pneumocytes in that the neoplastic cells express cytokeratin 7 , but more importantly, thyroid transcription factor 1 (TTF1), surfactant apoprotein, and Napsin A. Adenocarcinomas with extensive 


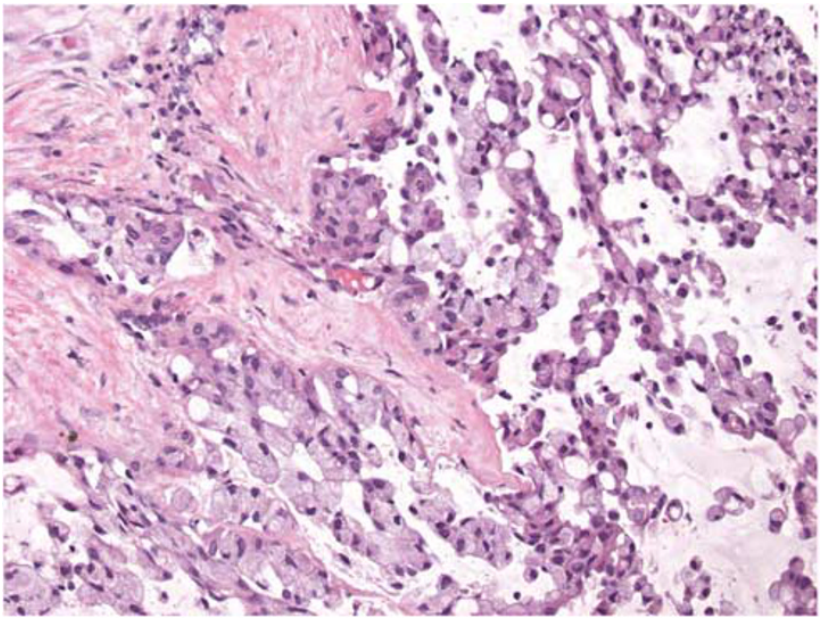

Figure 1 Epidermal growth factor receptor (EGFR)-mutated adenocarcinoma. Most EGFR-mutated adenocarcinomas are low grade, with prominent lepidic growth dominating a smaller invasive component (at right).

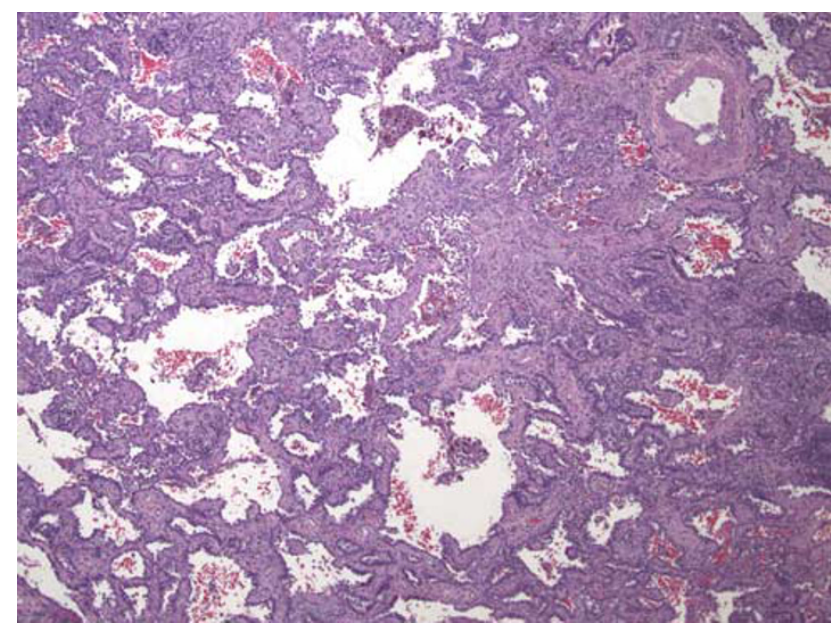

Figure 2 Epidermal growth factor receptor (EGFR)-mutated adenocarcinoma. The growth of these adenocarcinomas along pre-existing alveolar septa is seen at left, whereas invasion of lung with an associated desmoplastic inflammatory reaction is seen at right.

mucinous and goblet cell differentiation rarely have EGFR mutations, more commonly demonstrating K-RAS alterations. ${ }^{21}$

In the assessment of EGFR mutations, several approaches have been taken. Historically, immunoperoxidase stains for EGFR were applied to these lung adenocarcinomas. Although sensitive, panEGFR immunostaining is not specific and leads to a high false-positive rate, ${ }^{11,20,22,23}$ more recently, new antibodies specific to the mutations in exon 19 and 21 have been reported with high sensitivity and specificity, but these antibodies at the time of publication are not commercially available and therefore, cannot be used as a primary screening or diagnostic modality. ${ }^{24,25}$ Similarly, from a historical perspective, amplification of EGFR has been emphasized as one means of predicting response to

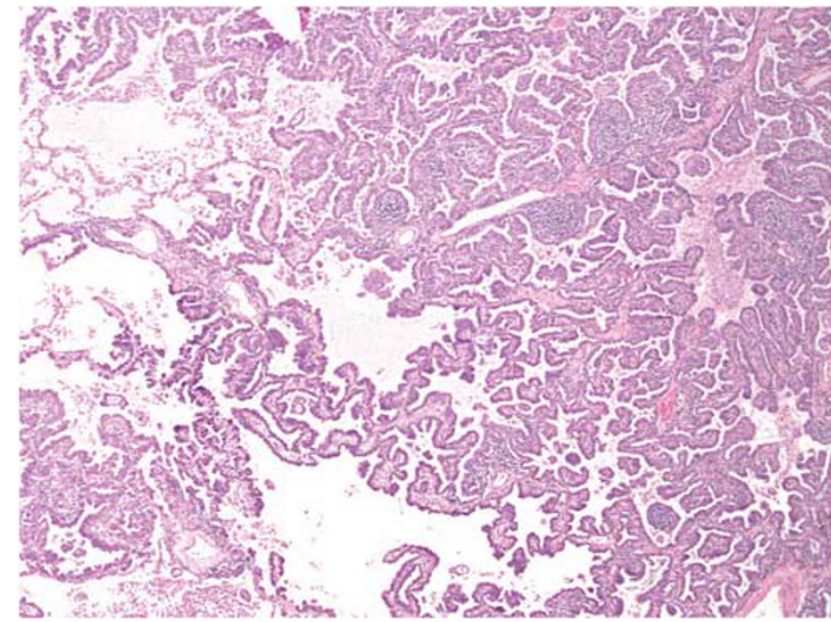

Figure 3 Epidermal growth factor receptor (EGFR)-mutated adenocarcinoma. These adenocarcinomas often have prominent papillary and micropapillary elements.

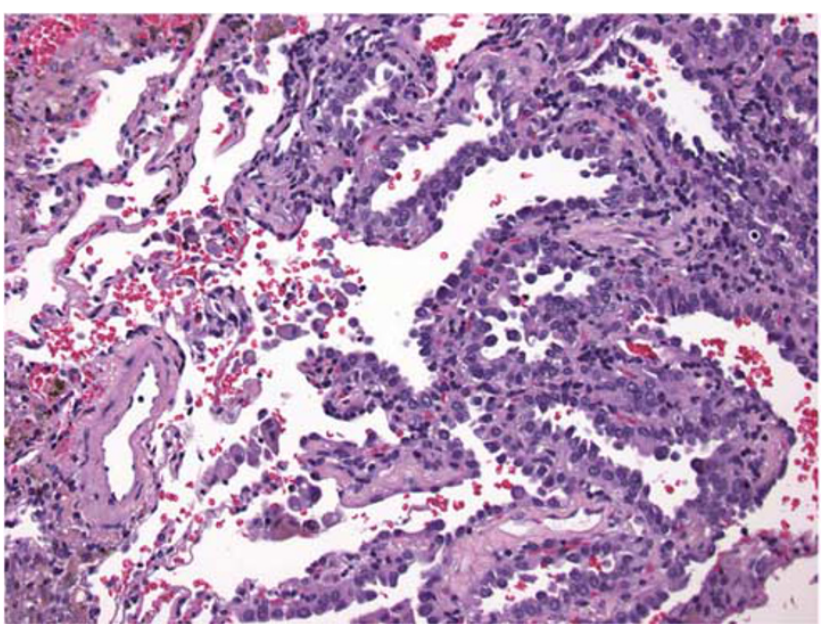

Figure 4 Epidermal growth factor receptor (EGFR)-mutated adenocarcinoma. Cytologically, the cells appear to be nonmucinous hobnail cells with Clara cell and Type II pneumocyte differentiation.

EGFR interventional therapy. Although amplification has been shown to predict a better overall survival after treatment with Gefitinib, the vast majority of literature argues that mutational analysis through DNA sequencing analysis of EGFR receptor, particularly focused on exons 19 and 21, offers much better predictability. ${ }^{4,9,17,24}$ The response to EGFR amplification is often explained by the fact that mutations in the EGFR are associated with gene amplification, and because wild-type adenocarcinomas have been shown to have a $10-20 \%$ response rate to tyrosine kinase inhibitor therapy as well. With mutations of exon 19 and 21, the therapeutic response is seen in over $85 \%$ of cases although many times it is short-lived, as other mutations occur in the EGFR receptor, particularly resistance mutations in exon 18. In those instances where recurrence or 


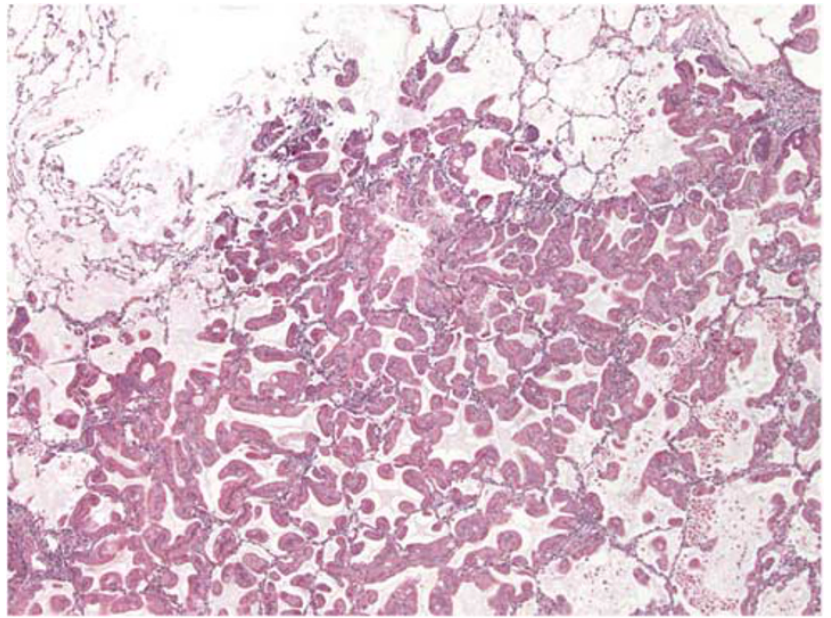

Figure 5 Mucinous adenocarcinoma. Mucinous or goblet cell differentiation in well-differentiated lepidic-predominant adenocarcinoma is unlikely to have epidermal growth factor receptor (EGFR) mutations; KRAS mutations predominate in this subgroup.

enlargement of a tumor mass on anti-EGFR therapy occurs, additional sequencing of exons 18 and 20 could help document mutations that confer resistance to tyrosine kinase inhibitors. Interestingly, the development of such mutations has been associated with rather prolonged and increased survival as compared with those tumors that progress without documentable resistance mutations. ${ }^{26}$

As noted before, the success of tyrosine kinase inhibitors in the treatment of EGFR-mutated lung cancers is measured in progression-free survival. In most cases, such survival is enhanced somewhere between 4 and 10 months; an effect on long-term survival has not been adequately documented. ${ }^{4,27}$

\section{EML4-ALK non-small cell carcinoma}

As the recognition of $A L K$-positive anaplastic lymphomas, translocations of this gene at the 2 p23 location has been of great interest, resulting particularly in the recognition that many inflammatory myofibroblastic tumors also have this translocation. The $A L K$ gene stimulates cellular proliferation, and is also thought to inhibit apoptosis as the $A L K$-gene inhibitors have augmented apoptosis of tumor cells and tumor shrinkage in vitro. In particular, new drugs such as Crizotinib/PF-2341066 have elicited a significant radiographic response in patients with $A L K$ gene mutations. In non-small cell carcinoma, it was initially recognized in Asian studies that the $A L K$ gene in some lung cancers underwent an inversion or translocation, with many different permutations, but most often as an inversion with the EML4-ALK. ${ }^{28-36}$

When studies were done looking at this translocation, between 4 and $8 \%$ of lung carcinoma showed this rearrangement. When the patient population with this abnormality was analyzed, it demonstrated

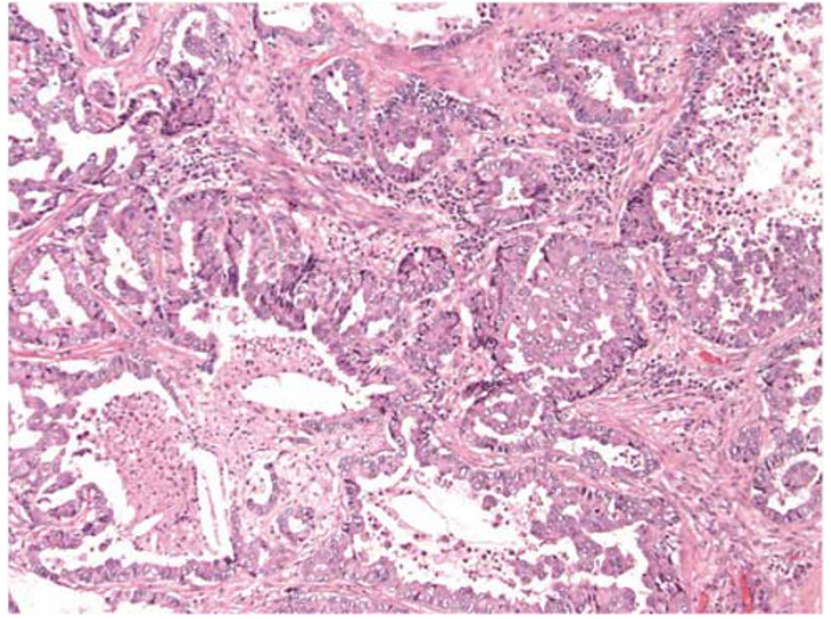

Figure 6 Anaplastic lymphoma kinase gene $(A L K)$-translocated adenocarcinoma. Most ALK translocated carcinomas are high grade, with solid and acinar growth often with extensive necrosis.

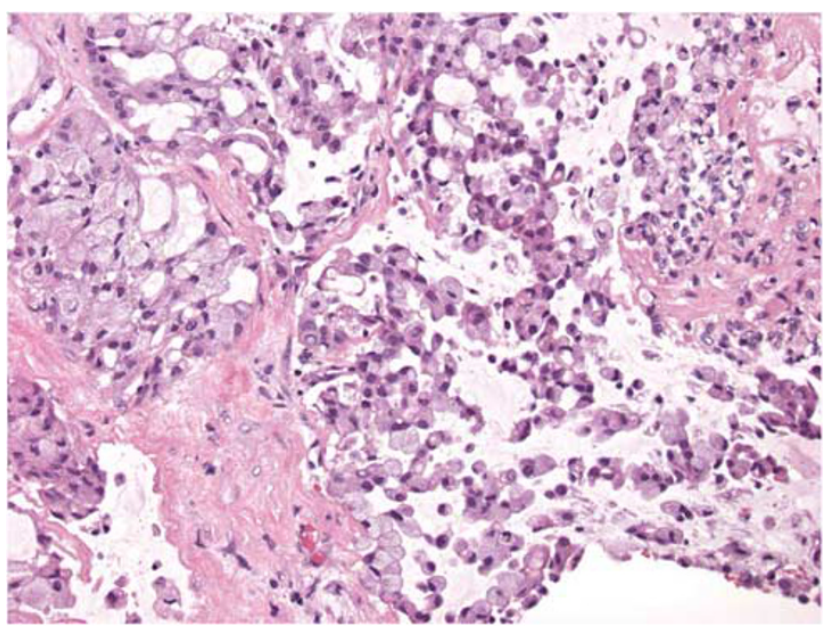

Figure 7 Anaplastic lymphoma kinase gene $(A L K)$-translocated adenocarcinoma. In a significant percentage of cases, these adenocarcinomas show mucinous and signet ring differentiation.

a predisposition to younger individuals in the 40-60 years age range, particularly men, with most having a light or absent cigarette-smoking history. ${ }^{32,37,38}$ In contrast to adenocarcinomas with EGFR mutations, patients with ALK rearranged tumors presented with high-stage disease. When such tumors were assessed histologically, most of these tumors were adenocarcinomas with a small percentage showing a squamous morphology. These adenocarcinomas were poorly differentiated ones often characterized by solid or acinar growth, often with broad regions of necrosis, and often accompanied by prominent host lymphocytic and stromal reaction $^{30,32}$ (Figures 6 and 7). Interesting, the majority of these adenocarcinomas expressed mucin, and somewhere between 30 and $50 \%$ of these, tumors showed signet ring differentiation. ${ }^{38}$ Immunohistochemical analysis showed terminal reserve unit 
cell phenotype, with TTF-1, surfactant apoprotein, and Napsin A positivity.

ALK-rearranged lung carcinomas have been of great interest recently, because of their putative response to new anti-ALK drugs, particularly Crizotinib. ${ }^{35,38}$ In patients with the oncogenic inversion of EML4 and ALK, an overall response rate of $57 \%$ was identified in high-stage lung adenocarcinoma after 6 months of therapy. ${ }^{38}$ Over $70 \%$ of patients showed an estimated 6 months progression-free survival, in contrast with a normal expectation of between 10 and $30 \%$. Such a dramatic response with tumor shrinkage and/or stable disease as a result of inhibition of ALK by Crizotinib has made identification of this variant of lung carcinoma of high importance.

In the assessment of lung cancers for ALK rearrangement, conventional studies using immunohistochemical stains for ALK 1 alterations have been characterized by mixed, often conflicting results. ${ }^{28,31}$ The majority of reports of immunostaining and lung cancer for ALK protein have shown that current antibodies used for ALK lymphoma diagnosis have low sensitivity, but high specificity. More recently, new antibodies have been described that have higher sensitivity and a higher specificity for $A L K$ gene expression in lung adenocarcinoma, but once again, the commercial availability of these antibodies has been very restricted. ${ }^{31}$ Consequently, utilizing these antibodies, eg, D5F3 is at the time of this publication, of uncertain future. Similarly, although DNA sequencing is preferred in the assessment of EGFR mutations, it is not the preferred method for analyzing ALK rearrangements, because the huge number $(>11)$ of partners of ALK translocation, as the association of these translocations with chromosome loss, make PCR assessment extremely difficult and laborious (eg, huge number of primer sets are required). Furthermore, the clinical significance of some fusion products is unclear. ${ }^{11,32}$ Consequently, in this reviewer's opinion, the best way of assessing ALK rearrangements is through fluorescence in situ hybridization studies. As the most common alteration is an inversion at the 2P23 location, the identification of rearrangements is extremely tricky, because one needs to carefully assess the distance between the two probe signals in a break-apart probe. Such assays are more easily identified when there is a chromosomal deletion or translocation to other chromosomes than chromosome number 2 . Controversy has also existed as to the diagnostic percentage of translocated cells that reflect a clinical response to Crizotinib therapy, eg, $15 \%$ is current recommendation. ${ }^{32}$

\section{BRAF-mutated lung adenocarcinoma}

Mutations of the RAF/mitogen-activated protein kinase signaling pathway can involve BRAF and K-RAS. This pathway culminates in transcription of genes favoring proliferation and survival, and 3 and $8 \%$ of lung adenocarcinomas have BRAF mutations. ${ }^{39-41}$ Over $50 \%$ of these mutations involve V600E, whereas lesser mutations are found at the G469A and G594G sites. B-RAF mutations are exclusive of EGFR, ALK and K-RAS mutations, and in contrast to EGFR and ALK mutations, are often seen in cigarette smokers. In studies, B-RAF mutations occur more typically in elderly individuals with a cigarette smoking history, and have an equal sex distribution, with some studies showing a subtle predilection for women. ${ }^{39,41}$

B-RAF mutations are most common in adenocarcinomas of the lung, particularly poorly differentiated adenocarcinomas with acinar and solid growth ${ }^{41}$ (Figures 8 and 9). As with EGFR-mutated adenocarcinomas, there is a high incidence of papillary and micropapillary growth, and mucin production,

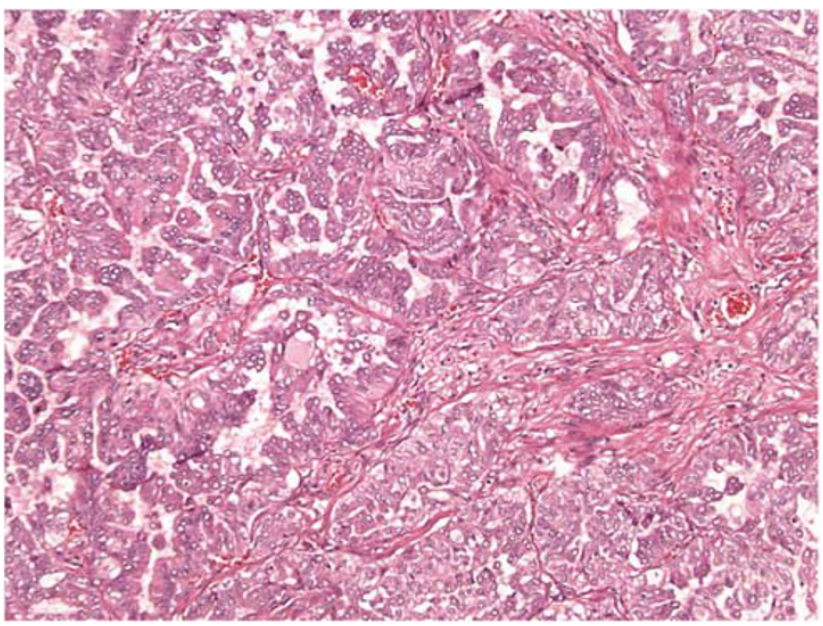

Figure 8 BRAF-mutated adenocarcinoma. Most of these adenocarcinomas are high grade, with solid, acinar, and papillary growth.

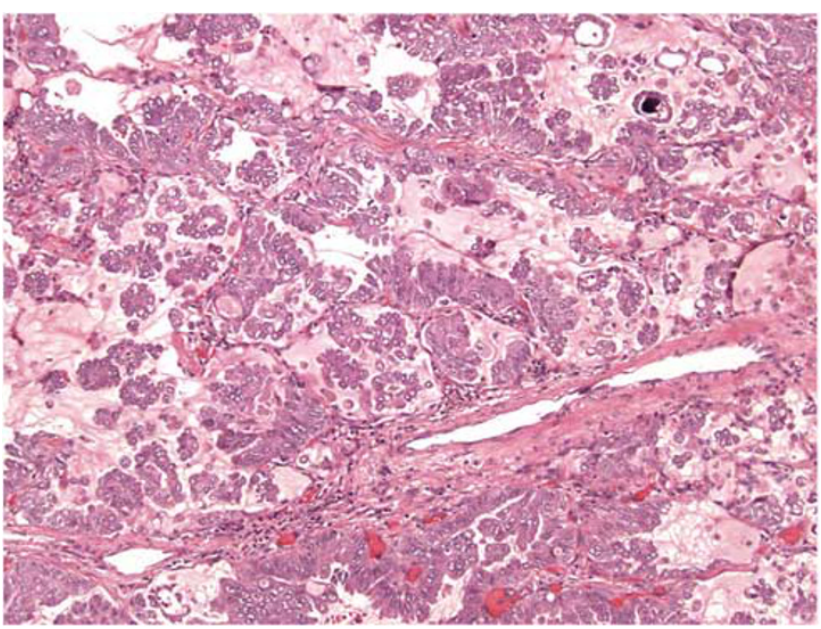

Figure 9 BRAF-mutated adenocarcinoma. These adenocarcinomas are heterogeneous and may have papillary, neuropapillary, and lepidic growth, often with mucin-secreting epithelium. 
although signet ring differentiation is not a prominent feature. Patients with BRAF mutations appear to present at higher stage, and this holds true even in clinically resected low-stage lung adenocarcinomas in which BRAF mutations are tied to high intralobar metastases, with satellite nodules and unexpected nodal disease.

As noted previously, BRAF mutations most often occur at the V-600E site, in EXON 15. At present, there is no good immunohistochemical or fluorescence in situ hybridization assay for BRAF mutations, and such mutations need to be assessed by direct DNA sequencing. ${ }^{41}$

Because of success in the treatment of malignant melanomas with BRAF mutations, there is a perception that this subgroup of lung adenocarcinomas should be aggressively assessed for the identification of therapeutic agents targeting the BRAF pathway. Such agents such as PLX4032 may have a significant effect in Phase I studies of high-stage lung adenocarcinoma with BRAF mutation.

\section{Summary}

Lung carcinoma has been a death sentence for patients in the twentieth and twenty-first century. These patients tend to present with high-stage disease, and conventional chemoradiation therapy for such patients has resulted in only minimal increases in progression-free survival in between 5 and $15 \%$ of patients receiving such therapy. Molecular testing has identified a subgroup of patients, less than $50 \%$, which has specific genetic mutations for which new therapeutic agents are available. At present, most assessments of these agents have been focused on patients with highstage disease in Phase I trials, but these trials are extremely promising. This optimism may relate to the fact that these carcinomas are genetically 'simple,' in that they are dominated by a single molecular alteration. Nonetheless, in diagnostic surgical pathology, if we can identify these subgroups through histological evaluation and subsequent ancillary testing, the long-term prognosis of patients with lung adenocarcinoma may improve.

\section{Disclosure/conflict of interest}

The author declares no conflict of interest.

\section{References}

1 Taylor BS, Ladanyi M. Clinical cancer genomics: how soon is now? J Pathol 2011;223:318-326.

2 Al-Saad S, Al-Shibli K, Donnem T, et al. Clinical significance of epidermal growth factor receptors in non-small cell lung cancer and a prognostic role for HER2 gene copy number in female patients. J Thorac Oncol 2010;5:1536-1543.
3 Bryant CM, Albertus DL, Kim S, et al. Clinically relevant characterization of lung adenocarcinoma subtypes based on cellular pathways: an international validation study. PLoS One 2010;5:e11712.

4 Eberhard DA, Giaccone G, Johnson BE. Biomarkers of response to epidermal growth factor receptor inhibitors in Non-Small-Cell Lung Cancer Working Group: standardization for use in the clinical trial setting. J Clin Oncol 2008;26:983-994.

5 Jiang H. Overview of gefitinib in non-small cell lung cancer: an Asian perspective. Jpn J Clin Oncol 2009;39:137-150.

6 Maemondo M, Inoue A, Kobayashi K, et al. Gefitinib or chemotherapy for non-small-cell lung cancer with mutated EGFR. New Engl J Med 2010;362:2380-2388.

7 Marks JL, Broderick S, Zhou Q, et al. Prognostic and therapeutic implications of EGFR and KRAS mutations in resected lung adenocarcinoma. J Thorac Oncol 2008;3:111-116.

8 Pao W, Wang TY, Riely GJ, et al. KRAS mutations and primary resistance of lung adenocarcinomas to gefitinib or erlotinib. PLoS Med 2005;2:e17.

9 Pirker R, Herth FJ, Kerr KM, et al. Consensus for EGFR mutation testing in non-small cell lung cancer: results from a European workshop. J Thorac Oncol 2010;5:1706-1713.

10 Travis WD, Brambilla E, Noguchi M, et al. International Association for the Study of Lung Cancer/ American Thoracic Society/European Respiratory Society International Multidisciplinary Classification of Lung Adenocarcinoma. J Thorac Oncol 2011;6: 244-285.

11 Dacic S. EGFR assays in lung cancer. Adv Anat Pathol 2008;15:241-247.

12 Monaco SE, Nikiforova MN, Cieply K, et al. A comparison of EGFR and KRAS status in primary lung carcinoma and matched metastases. Hum Pathol 2010;41:94-102.

13 Smouse JH, Cibas ES, Janne PA, et al. EGFR mutations are detected comparably in cytologic and surgical pathology specimens of nonsmall cell lung cancer. Cancer Cytopathol 2009;117:67-72.

14 Nakano H, Soda H, Takasu M, et al. Heterogeneity of epidermal growth factor receptor mutations within a mixed adenocarcinoma lung nodule. Lung Cancer 2008;60:136-140.

15 Zhong WZ, Wu YL, Yang XN, et al. Genetic evolution of epidermal growth factor receptor in adenocarcinoma with a bronchioloalveolar carcinoma component. Clin Lung Cancer 2010;11:160-168.

16 Gow CH, Chang YL, Hsu YC, et al. Comparison of epidermal growth factor receptor mutations between primary and corresponding metastatic tumors in tyrosine kinase inhibitor-naive non-small-cell lung cancer. Ann Oncol 2009;20:696-702.

17 Li AR, Chitale D, Riely GJ, et al. EGFR mutations in lung adenocarcinomas: clinical testing experience and relationship to EGFR gene copy number and immunohistochemical expression. J Mol Diagn 2008;10: 242-248.

18 Zakowski MF, Hussain S, Pao W, et al. Morphologic features of adenocarcinoma of the lung predictive of response to the epidermal growth factor receptor kinase inhibitors erlotinib and gefitinib. Arch Pathol Lab Med 2009;133:470-477.

19 Motoi N, Szoke J, Riely GJ, et al. Lung adenocarcinoma: modification of the 2004 WHO mixed subtype to 
include the major histologic subtype suggests correlations between papillary and micropapillary adenocarcinoma subtypes, EGFR mutations and gene expression analysis. Am J Surg Pathol 2008;32: 810-827.

20 Borczuk AC. Micropapillary histology: a frequent morphology of mutation-associated lung adenocarcinoma? Am J Clin Pathol 2009;131:615-617.

21 Dacic S, Shuai Y, Yousem S, et al. Clinicopathological predictors of EGFR/KRAS mutational status in primary lung adenocarcinomas. Mod Pathol 2010;23:159-168.

22 Brevet M, Arcila M, Ladanyi M. Assessment of EGFR mutation status in lung adenocarcinoma by immunohistochemistry using antibodies specific to the two major forms of mutant EGFR. J Mol Diagn 2010;12: 169-176.

23 Cappuzzo F. EGFR FISH versus mutation: different tests, different end-points. Lung Cancer 2008;60: 160-165.

24 Pan Q, Pao W, Ladanyi M. Rapid polymerase chain reaction-based detection of epidermal growth factor receptor gene mutations in lung adenocarcinomas. J Mol Diagn 2005;7:396-403.

$25 \mathrm{Yu}$ J, Kane S, Wu J, et al. Mutation-specific antibodies for the detection of EGFR mutations in non-small-cell lung cancer. Clin Cancer Res 2009;15:3023-3028.

26 Sequist LV, Waltman BA, Dias-Santagata D, et al. Genotypic and histological evolution of lung cancers acquiring resistance to EGFR inhibitors. Sci Transl Med 2011;3:75ra26.

27 Yoo SB, Chung JH, Lee HJ, et al. Epidermal growth factor receptor mutation and p53 overexpression during the multistage progression of small adenocarcinoma of the lung. J Thorac Oncol 2010;5:964-969.

28 Boland JM, Erdogan S, Vasmatzis G, et al. Anaplastic lymphoma kinase immunoreactivity correlates with ALK gene rearrangement and transcriptional up-regulation in non-small cell lung carcinomas. Hum Pathol 2009;40:1152-1158.

29 Inamura K, Takeuchi K, Togashi Y, et al. EML4-ALK lung cancers are characterized by rare other mutations, a TTF-1 cell lineage, an acinar histology, and young onset. Mod Pathol 2009;22:508-515.
30 Martelli MP, Sozzi G, Hernandez L, et al. EML4-ALK rearrangement in non-small cell lung cancer and non-tumor lung tissues. Am J Pathol 2009;174: $661-670$.

31 Mino-Kenudson M, Chirieac LR, Law K, et al. A novel, highly sensitive antibody allows for the routine detection of ALK-rearranged lung adenocarcinomas by standard immunohistochemistry. Clin Cancer Res 2010;16:1561-1571.

32 Rodig SJ, Mino-Kenudson M, Dacic S, et al. Unique clinicopathologic features characterize ALK-rearranged lung adenocarcinoma in the western population. Clin Cancer Res 2009;15:5216-5223.

33 Sasaki T, Rodig SJ, Chirieac LR, et al. The biology and treatment of EML4-ALK non-small cell lung cancer. Eur J Cancer 2010;46:1773-1780.

34 Shaw AT, Yeap BY, Mino-Kenudson M, et al. Clinical features and outcome of patients with non-small-cell lung cancer who harbor EML4-ALK. J Clin Oncol 2009;27:4247-4253.

35 Takeuchi K, Choi YL, Togashi Y, et al. KIF5B-ALK, a novel fusion oncokinase identified by an immunohistochemistry-based diagnostic system for ALK-positive lung cancer. Clin Cancer Res 2009;15:3143-3149.

36 Yoshida A, Tsuta K, Nakamura H, et al. Comprehensive histologic analysis of ALK-rearranged lung carcinomas. Am J Surg Pathol 2011;35:1226-1234.

37 Kotsakis A, Yousem S, Gadgeel SM. Is histologic subtype significant in the management of NSCLC? Open Lung Cancer J 2010;3:65-71.

38 Kwak EL, Bang YJ, Camidge DR, et al. Anaplastic lymphoma kinase inhibition in non-small-cell lung cancer. N Engl J Med 2010;363:1693-1703.

39 Paik PK, Arcila ME, Fara M, et al. Clinical characteristics of patients with lung adenocarcinomas harboring BRAF mutations. J Clin Oncol 2011;29:2046-2051.

40 Schmid K, Oehl N, Wrba F, et al. EGFR/KRAS/BRAF mutations in primary lung adenocarcinomas and corresponding locoregional lymph node metastases. Clin Cancer Res 2009;15:4554-4560.

41 Yousem SA, Nikiforova M, Nikiforov Y. The histopathology of BRAF-V600E-mutated lung adenocarcinoma. Am J Surg Pathol 2008;32:1317-1321. 